\title{
Individuelle Beschäftigungsfähigkeit: Konzept, Operationalisierung und erste Ergebnisse
}

\author{
Martin Brussig \\ Matthias Knuth
}

Die Erhöhung der Beschäftigungsfähigkeit ist Kernbestandteil der europäischen Beschäftigungsstrategie, doch die meisten Studien hierzu sind über konzeptionelle Diskussionen nicht hinausgekommen. Evaluationen in der Arbeitsmarktpolitik erfordern die Entwicklung empirisch messbarer Indikatoren von Beschäftigungsfähigkeit. Dieser Aufsatz stellt empirische Ergebnisse zur Beschäftigungsfähigkeit von Arbeitslosengeld (ALG)-II-Beziehern vor. Sie erlauben Rückschlüsse auf individuelle Integrationsprobleme und strukturelle Beschäftigungsbarrieren bei ALG-II-Empfängern. Die Ergebnisse stehen nur teilweise im Einklang mit gängigen Annahmen der ,aktivierenden“ Arbeitsmarktpolitik.

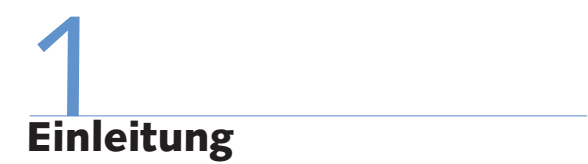

Evaluationen in der Arbeitsmarktpolitik erfordern die Entwicklung empirisch messbarer Indikatoren von Beschäftigungsfähigkeit, deren Erhöhung seit nun schon mehr als zehn Jahren Ziel der Arbeitsmarktpolitik ist. Doch erst in jüngster Zeit wurden in der deutschen Arbeitsmarktforschung Ansätze zur Operationalisierung des Konzepts von Beschäftigungsfähigkeit - verstanden insbesondere als Fähigkeit, eine Arbeit aufzunehmen, aber auch, sie auszuweiten und aufrechtzuerhalten entwickelt (Deeke/Kruppe 2003; Söstra et al. 2005; Apel/Fertig 2008). Dabei traten Probleme auf, die bislang nicht befriedigend gelöst werden konnten: Deeke/Kruppe (2003) sehen Beschäftigungsfähigkeit als entweder durch den outcome oder durch einen arbeitsmarktpolitischen input definiert: „Realisierte Beschäftigungsfähigkeit“ liegt demnach vor, wenn eine Beschäftigung aufgenommen wurde, , hypothetische Beschäftigungsfähigkeit" ergibt sich aus der erfolgreichen Teilnahme an einer Weiterbildungsmaßnahme (ebd., S. 11). Doch dass die Aufnahme einer Beschäftigung auf die Fähigkeit dazu schließen lässt, ist tautologisch, während andererseits die positive Wirkung einer Weiterbildung auf diese Fähigkeit empirisch zu prüfen und nicht einfach vorauszusetzen wäre. Alternativ wurde in einer neueren Evaluation von Ar- beitsbeschaffungsmaßnahmen Beschäftigungsfähigkeit in erster Linie als subjektives Wohlbefinden von den Teilnehmern direkt erfragt (Söstra et al. 2005). Allerdings fehlte hier der Nachweis des Zusammenhangs der Selbsteinstufung mit realen Beschäftigungspotenzialen. Schließlich haben Apel/Fertig (2009) für die Bestimmung von Beschäftigungsfähigkeit auf individueller Ebene einen wichtigen Beitrag geleistet, indem sie Gruppen von Items zur Erfassung von Dimensionen der Beschäftigungsfähigkeit empirisch überprüft haben. ${ }^{1}$ Auf dem in dieser Arbeit entwickelten Set von Indikatoren bauen wir auf. Die Grenze des Beitrages von Apel/Fertig (2009) ist darin zu sehen, dass sie zwar aus einer Fülle von getesteten Items ein effizientes Itemset zur Erfassung individueller Beschäftigungsfähigkeit bestimmt haben, doch standen in dieser Vorstudie keine Kontextvariablen zur Verfügung, um den Einfluss struktureller Bedingungen gegenüber der individuellen Beschäftigungsfähigkeit für eine Beschäftigungsaufnahme abzuwägen.

In diesem Beitrag gehen wir auf Grundlage einer umfangreichen Befragung von ALG-II-Beziehern und-Bezieherinnen erstens darauf ein, ob ein daraus gewonnenes Konstrukt individueller Beschäftigungsfähigkeit zur Erklärung von Erwerbsintegration überhaupt empirisch in signifikanter Weise beiträgt. Wir analysieren zweitens, welche Dimensionen von Beschäftigungsfähigkeit sich als besonders einflussreich für den Übergang in eine Beschäftigung erweisen, und drittens wird diskutiert, ob es strukturelle Unterschiede in der individuellen Beschäftigungsfähigkeit zwischen Personen aus unterschiedlichen arbeitsmarktpolitischen Zielgruppen gibt.

Bevor wir Ergebnisse zu diesen empirisch orientierten Fragen präsentieren, erinnern wir zunächst an wesentliche Aspekte der konzeptionellen Diskussionen um den Begriff der Beschäftigungsfähigkeit (Abschnitt 2). Wir stellen dann unser empirisches Konzept und die verwendete Datengrundlage vor (Abschnitt 3) und diskutieren die empirischen Ergebnisse (Abschnitt 4), die wir im Fazit (Abschnitt 5) auch im Lichte gängiger arbeitsmarktpolitischer Annahmen zusammenfassen.

\footnotetext{
Grundlage war eine telefonische Befragung von je 2.000 ALG- bzw. ALG-II-Beziehenden, die - unter Beteiligung der Autoren des vorliegenden Aufsatzes - als "Vorstudie" für eine breit angelegte Befragung von ALG-II-Beziehenden fungierte, aus der wir hier berichten.
}

Martin Brussig, Dr. wissenschaftlicher Mitarbeiter am Institut Arbeit und Qualifikation (IAQ) der Universität Duisburg-Essen. Arbeitsschwerpunkte: Erwerbstätigkeit und demografischer Wandel; Arbeitsmarktpolitik. e-mail: martin.brussig@uni-due.de Matthias Knuth, Prof. Dr., Abteilungsleiter am IAQ. Arbeitsschwerpunkte: Arbeits- und Sozialpolitik, Erwerbssystem. e-mail: matthias.knuth@uni-due.de 


\section{Abb. 1: Komponenten und Dimensionen von individueller Beschäftigungsfähigkeit}

\section{Anmerkungen zum Konzept von Beschäftigungsfähigkeit}

Das Konzept der Beschäftigungsfähigkeit hat in den letzten Jahren eine bemerkenswerte Karriere gemacht. Ein wesentlicher Impuls kam aus der Arbeitsmarktpolitik (McQuaid/Lindsay 2005), denn in der seit Mitte der 1990er Jahre aufgekommenen „aktivierenden“ Arbeitsmarktpolitik wird die Eigenverantwortung der Arbeitsmarktakteure, insbesondere der Arbeitsuchenden, deutlich stärker betont als in der „aktiven“ Arbeitsmarktpolitik des fordistischen Industriezeitalters. Die Erhöhung individueller Beschäftigungsfähigkeit ist daher auch zentraler Bestandteil der Europäischen Beschäftigungsstrategie (Europäische Kommission 1997; Lefresne 1999) und in Deutschland gesetzliches Ziel der Arbeitsförderung nach dem SGB III. ${ }^{2}$

Die Wurzeln des Konzeptes „Beschäftigungsfähigkeit“ gehen aber tiefer und reichen bis an den Beginn sozialstaatlicher Leistungen wegen Erwerbslosigkeit und damit bis an die Wende vom 19. zum 20. Jahrhundert zurück (Gazier 1998). Der Bezug einer Unterstützung wegen Erwerbslosigkeit aufgrund von Invalidität setzt „Beschäftigungsunfähigkeit" voraus. Aufgrund der erforderlichen eindeutigen Entscheidung, die vornehmlich unter medizinischen Gesichtspunkten getroffen wird, spricht Gazier hier vom "dichotomen“ Konzept von Beschäftigungsfähigkeit. Dieses Konzept ist auch heute aktuell, denn die "Grundsicherung für Arbeitsuchende“ können nur Personen beanspruchen, die „erwerbsfähig“ sind ( $\$ 7$, Abs. 1, Nr. 2 SGB II; vgl. auch Fußnote 2).

In der arbeitsmarkttheoretischen Debatte werden heute überwiegend zwei Positionen gegeneinander in Stellung gebracht: das individuelle und das interaktive Konzept von Beschäftigungsfähigkeit (z. B. Promberger et al. 2008). Das Konzept der individuellen Beschäftigungsfähigkeit entstand historisch in den 1950er Jahren und bezog sich beispielsweise auf gesundheitlich Benachteiligte. In dem Maße, wie die strukturellen Aspekte von Arbeitslosigkeit und damit auch die individuellen Voraussetzungen für eine Beschäftigung im Verhältnis zu den von den Arbeitgebern gestellten Anforderungen ins Blickfeld traten, weitete sich der Adressatenkreis auf weite-

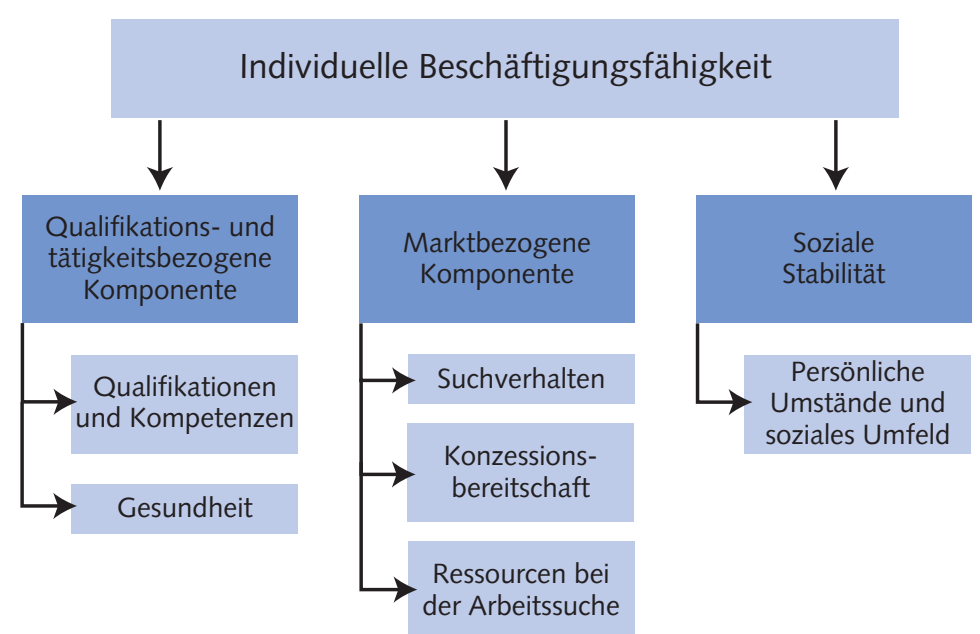

Quelle: Darstellung der Autoren

WSI MITTEILUNGEN

re „Problemgruppen“ aus. Mit dem Übergang zur aktivierenden Arbeitsmarktpolitik wird in der individuellen Beschäftigungsfähigkeit eine allgemeine, nicht auf Problemgruppen beschränkte, individuelle Voraussetzung zur Überwindung von Arbeitslosigkeit gesehen.

Gegen das Konzept der individuellen Beschäftigungsfähigkeit wird vor allem vorgebracht, dass die Arbeitsmarktchancen stets im Zusammenhang mit der quantitativen und qualitativen Arbeitsnachfrage sowie den institutionellen Bedingungen des Arbeitsmarktes gesehen werden müssen. Sie auf Dispositionen und Ressourcen von arbeitsuchenden Individuen zu reduzieren, laufe auf eine unangemessene Schuldzuweisung hinaus. Dies wird im Konzept der interaktiven Beschäftigungsfähigkeit deutlich (Gazier 1998), welches betont, „, that the employability of the individual is relative to the employability of others and the opportunities, institutions and rules that govern the labour market" (McQuaid/Lindsay 2005, S. 201). Hier geht es nicht nur um den einfachen Sachverhalt, dass die Arbeitsmarktchancen im Aufschwung steigen oder von der regionalen Arbeitsmarktlage abhängen. Angesprochen sind auch betriebliche Strategien. Wenn beispielsweise Betriebe ältere Bewerber für Neueinstellungen grundsätzlich nicht in Betracht ziehen (Bellmann et al. 2007), tragen die individuellen Voraussetzungen von älteren Bewerbern wenig zu ihren Jobchancen bei. Auch die institutionellen Bedingungen des Arbeitsmarktes beeinflussen, wie schnel sich eine wachsende Arbeitsnachfrage in einer Zunahme der Beschäftigung niederschlägt und ob diese zusätzliche Beschäftigung aus dem Kreis der bislang Arbeitslosen kommt. So weit stellt dies jedoch keine Gegenposition zum Konzept der individuellen Beschäftigungsfähigkeit dar, das nicht den Anspruch erhebt, Beschäftigungschancen insgesamt zu erklären, sondern vielmehr die individuelle Fähigkeit zur Beschäftigungsaufnahme thematisiert. Der Einfluss struktureller Gegebenheiten ist vielmehr in ein empirisches Modell einzubeziehen.

Individuelle Beschäftigungsfähigkeit ist ein mehrdimensionales Konstrukt. Über die unterschiedlichen Komponenten von Beschäftigungsfähigkeit und ihre Beziehungen untereinander gibt es keine einhellige Auffassung (vgl. die Literaturübersicht bei Blancke et al. 2000 und Apel 2006). Als gemeinsamer Nenner lassen sich eine qua-

2 „Die Leistungen der Arbeitsförderung sollen insbesondere (...) die individuelle Beschäftigungsfähigkeit durch Erhalt und Ausbau von Kenntnissen, Fähigkeiten und Fertigkeiten fördern" (§1, Abs. 2 SGB III). - Analog heißt es im SGB II: „Die Leistungen der Grundsicherung sind insbesondere darauf auszurichten, dass ... 2. die Erwerbsfähigkeit des Hilfebedürftigen erhalten, verbessert oder wiederhergestellt wird" (§ 1, Abs. 1, Satz 4 SGB II). Diese Formulierung ist paradox, weil es nach der Abgrenzung des Rechtskreises des SGB II in §7. Abs. 1 SGB II darin eigentlich keine Personen geben kann, deren Erwerbsfähigkeit "wiederhergestellt " werden müsste. Folglich ist davon auszugehen, dass "Erwerbsfähigkeit" hier das Gleiche meint wie "Beschäftigungsfähigkeit" . 


\begin{tabular}{|c|c|c|}
\hline \multicolumn{3}{|c|}{ Übersicht 1: Indikatoren für Beschäftigungsfähigkeit } \\
\hline Dimension & Indikatoren & Bemerkung \\
\hline \multicolumn{3}{|c|}{ Qualifikations- und tätigkeitsbezogene Komponente } \\
\hline \multicolumn{3}{|c|}{ Qualifikationen und Kompetenzen } \\
\hline $\begin{array}{l}\text { Formal- } \\
\text { qualifikation }\end{array}$ & $\begin{array}{l}\text { Niedrig (ohne Abschluss) } \\
\text { Mittel (betrieblich Ausbildung, Meister, Berufs-/Fachakademie) } \\
\text { Hoch (Fachhochschul-/Hochschulabschluss) }\end{array}$ & \\
\hline $\begin{array}{l}\text { Basis- } \\
\text { kompetenzen }\end{array}$ & $\begin{array}{l}\text { Lesen und Schreiben } \\
\text { Rechnen } \\
\text { E-mail, Internet }\end{array}$ & $\begin{array}{l}\text { Selbsteinschätzung } \\
\text { mit Schulnoten }\end{array}$ \\
\hline $\begin{array}{l}\text { Sozial- } \\
\text { kompetenzen }\end{array}$ & $\begin{array}{l}\text { Selbsteinschätzungen zu: } \\
\text { - Ich arbeite gut und gern mit anderen zusammen } \\
\text { - Es macht mir Spaß, mich in neue Aufgaben einzuarbeiten } \\
\text { - Ich setze mir Ziele u. verwirkliche sie ohne Anstöße von } \\
\text { außen } \\
\text { - Ich arbeite genau und berücksichtige dabei alle } \\
\text { erforderlichen Aspekte }\end{array}$ & Index von $0 \ldots 4$ \\
\hline Persönlichkeit & $\begin{array}{l}\text { Selbsteinschätzungen zu: } \\
\text { - Treffe mich häufig mit Freunden und Bekannten } \\
\text { - Bin häufig lustlos und niedergeschlagen } \\
\text { - Ich weiß, dass ich gebraucht werde } \\
\text { - Ich übernehme gern Verantwortung für das, was ich tue }\end{array}$ & Index von $0 \ldots 4$ \\
\hline Gesundheit & $\begin{array}{l}\text { Allgemeinbefinden } \\
\text { Gesundheitliche tägliche Arbeitsfähigkeit in Stunden }\end{array}$ & $\begin{array}{l}\text { Index mit } 5 \text { Auspräg. } \\
\text { aus beiden Indikatoren }\end{array}$ \\
\hline \multicolumn{3}{|c|}{ Marktbezogene Komponente } \\
\hline Suchverhalten & $\begin{array}{l}\text { Suchwege } \\
\text { Bewerbungsgespräche in letzten } 6 \text { Monaten }\end{array}$ & Jeweils Anzahl \\
\hline $\begin{array}{l}\text { Konzessions- } \\
\text { bereitschaft }\end{array}$ & $\begin{array}{l}\text { Langer Arbeitsweg } \\
\text { Ungünstige Arbeitszeit } \\
\text { Unangenehme Arbeitsbedingungen } \\
\text { Umzug } \\
\text { Niedriges Einkommen }\end{array}$ & $\begin{array}{l}\text { Jeweils } 1 \text { für ,"auf } \\
\text { jeden Fall“/,"event.", } \\
0 \text { für ",wahrscheinlich } \\
\text { nicht" "/, auf keinen } \\
\text { Fall“" }\end{array}$ \\
\hline $\begin{array}{l}\text { Individuelle } \\
\text { Ressourcen }\end{array}$ & $\begin{array}{l}\text { Führerschein (ohne Kfz) } \\
\text { Fahrzeug (u. Führerschein) } \\
\text { Selbsteinschätzungen zu: } \\
\text { - Wert der Qualifikationen } \\
\text { - Wert der Arbeitserfahrungen } \\
\text { - Hilfreiche Kontakte }\end{array}$ & $\begin{array}{l}\text { Jeweils Nein/Ja } \\
\text { Jeweils } 0 / 1 \text { für } \\
\text { positive/negative } \\
\text { Selbsteinschätzung }\end{array}$ \\
\hline \multicolumn{3}{|c|}{ Soziale Stabilität } \\
\hline $\begin{array}{l}\text { Persönliche } \\
\text { Umstände }\end{array}$ & $\begin{array}{l}\text { Unterstützungsbedarf: } \\
\text { - psychische Beratung } \\
\text { - Suchtberatung } \\
\text { - Schuldenberatung } \\
\text { - Betreuung minderjähriger Kinder } \\
\text { - Familiäre Konflikte }\end{array}$ & $\begin{array}{l}\text { Jeweils } 1 \text { wenn } \\
\text { Unterstützungsbedarf, } \\
\text { sonst } 0\end{array}$ \\
\hline & Netzwerk: Anzahl von unterstützenden Personen & Anzahl \\
\hline $\begin{array}{l}\text { Soziales } \\
\text { Umfeld }\end{array}$ & $\begin{array}{l}\text { Umfeld: Selbsteinschätzungen zu: } \\
\text { - Kenne viele mit ALG II } \\
\text { - Habe beruflich erfolgreiche Freunde } \\
\text { - Familie interessiert sich für mich }\end{array}$ & $\begin{array}{l}\text { Jeweils } 1 \text { bei } \\
\text { Zustimmung, sonst } 0\end{array}$ \\
\hline
\end{tabular}

lifikations- und tätigkeitsbezogene Komponente, eine marktbezogene Komponente sowie die Komponente sozialer Stabilität ausmachen, die sich zu insgesamt sechs Dimensionen ausdifferenzieren lassen ( $A b$ bildung 1). Die qualifikations- und tätigkeitsbezogene Komponente erfasst die Voraussetzungen, eine bestimmte Erwerbstätigkeit überhaupt dauerhaft und zur $\mathrm{Zu}$ friedenheit von Arbeit- oder Auftraggebern ausführen zu können, während die marktbezogene Komponente abbildet, inwiefern Arbeitsuchende in der Lage sind, potenzielle Arbeitgeber zu finden und von den eigenen Qualitäten zu überzeugen. Die Komponente sozialer Stabilität erfasst Persönlichkeitseigenschaften und persönliche Umstände, die für die Aufnahme von Er-

\section{Untersuchungskonzept und Datengrundlage}

Datengrundlage ist eine telefonische Befragung von ALG-II-Beziehenden, die im Zusammenhang mit der Evaluierung der Experimentierklausel nach $\$ 6 c$ SGB II durchgeführt wurde (ZEW et al. 2008). Befragt wurden jeweils 25.000 Personen zu zwei Zeitpunkten (Winter 2006/07 und Winter
2007/08), die entweder zu einem definierten Zeitpunkt im Spätsommer 2006 im Bestand der Leistungsbezieher waren („Bestandsstichprobe“; ca. $80 \%$ der Befragten) oder in zwei eng definierten Zeiträumen jeweils 2006 und 2007 in den Leistungsbezug eingetreten sind („Zugangsstichproben“; ca. $20 \%$ der Befragten). Unter den Befragten der ersten Welle konnten knapp 14.000 Personen auch zum zweiten Zeitpunkt befragt werden (Panelteilnehmer). Befragungsinhalte waren in erster Linie Erfahrungen der Leistungsbezieher mit den Grundsicherungsstellen und der Aktivierung, die Nutzung arbeitsmarktpolitischer Maßnahmen, Formen der Erwerbsintegration sowie Angaben zur Lebenssituation der Befragten und der zugehörigen Haushaltsmitglieder (Bedarfsgemeinschaft). Erhoben wurden aber auch die Dimensionen von individueller Beschäftigungsfähigkeit. Die entsprechenden Indikatoren, mit denen die Beschäftigungsfähigkeit operationalisiert wurde (Abbildung 1), sind in Übersicht 1 dargestellt. Entsprechend dem Untersuchungskontext stehen vor allem solche individuellen Eigenschaften im Mittelpunkt, deren Beeinflussung durch die Interventionen von Vermittlungsfachkräften und Fallmanagern nicht von vornherein unmöglich (wie z. B. das Alter) erscheint. Dass die erhobenen Items die entsprechenden Dimensionen abbilden, wurde in der „Vorstudie“ überprüft (vgl. Fußnote 1).

Für die hier vorgestellte Analyse wurde die Stichprobe eingeschränkt. Einbezogen wurden nur Personen des Panels, die nicht zwischen den beiden Befragungszeitpunkten durchgängig erwerbstätig waren. ${ }^{3}$ Aus dem Modell wurden Jugendliche und Auszubildende ausgeschlossen, weil sie zu groBen Anteilen unabhängig von ihrer Beschäftigungsfähigkeit in Zuständen der Nicht-Erwerbstätigkeit verbleiben (Schule, Ausbildung) oder dahin einmünden. Insgesamt gingen 8.814 Fälle in die Analyse ein.
3 Das Panel enthält auch Beschäftigte im aufstockenden Leistungsbezug. Soweit dieser Status dauerhaft war, bestand bei diesen Fällen keine Chance, eine erneute Beschäftigungsaufnahme zu beobachten. Auch kann bei "Aufstockern" keine Arbeitssuche vorausgesetzt werden, was zur Folge hat, dass die entsprechenden Indikatoren nicht verwendet werden können. Sie wurden deshalb aus den folgenden Analysen ausgeschlossen. Die Bildung eines Indikators der Beschäftigungsfähigkeit, der für Beschäftigte und Arbeitsuchende gleichermaßen anwendbar ist, ist eine noch ungelöste Aufgabe. 
Für Personen der Untersuchungsstichprobe wurde anhand ihrer individuellen Ressourcen und Dispositionen zum Zeitpunkt $\mathrm{t}_{1}$ unter Kontrolle von Kontext- und Kontrollvariablen regressionsanalytisch geschätzt, welchen Einfluss die einzelnen Dimensionen bzw. die sie abbildenden Indikatoren auf eine Beschäftigungsaufnahme bis zu einem späteren Zeitpunkt $\left(t_{2}\right)$ haben. ${ }^{4}$ Etwa ein Viertel der befragten Personen verzeichnete (mindestens) eine Beschäftigungsaufnahme (2.246 Personen). Die Koeffizienten, die sich für jeden einzelnen Indikator von Beschäftigungsfähigkeit aus der Regressionsschätzung ergeben, werden zu einem einzigen Wert verdichtet, indem die Koeffizienten mit der individuellen Ausprägung des betreffenden Merkmals multipliziert und anschließend für alle Indikatoren addiert werden. Die individuelle Ausprägung eines Indikators wird also mit dem Einfluss gewichtet, der ihm für die Beschäftigungsaufnahme im Durchschnitt aller Personen der Stichprobe zukommt. Auch für jene Personen, bei denen keine Beschäftigungsaufnahme beobachtet wurde, wird der individuelle Wert ihrer Beschäftigungsfähigkeit gebildet aus Indikatoren, gewichtet mit dem Einflussgewicht, das bei den Personen beobachtet wurde, die Beschäftigung aufnahmen. Da diese Indikatoren im Panel zu zwei Zeitpunkten erhoben wurden, ist es möglich, die Wirkung von , aktivierenden“ Interventionen auf die Veränderung der Beschäftigungsfähigkeit zu bestimmen - was allerdings nicht Gegenstand dieses Beitrags ist.

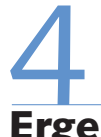

Ergebnisse

\subsection{INDIVIDUELLE BESCHÄFTIGUNGS- FÄHIGKEIT: EIN ERSTER TEST}

Wenn die Aufnahme einer Beschäftigung nicht im Zusammenhang stünde mit dem von uns gemessenen Konstrukt der individuellen Beschäftigungsfähigkeit, dann dürfte es keinen Unterschied in dem Indexwert für die Beschäftigungsfähigkeit zwischen denjenigen geben, die eine Beschäftigung gefunden haben, und jenen, die beschäftigungslos geblieben sind. Die Ergebnisse weisen aber einen deutlichen Unterschied in der durchschnittlichen Beschäftigungsfähigkeit beider Gruppen aus (Tabelle 1). Dies bestätigt die Annahme,

Tabelle 1: Indexwerte für Beschäftigungsfähigkeit für Personen mit bzw. ohne Aufnahme einer Beschäftigung

\begin{tabular}{lcc}
\hline & $\begin{array}{c}\text { Personen mit Aufnahme } \\
\text { einer Beschäftigung }\end{array}$ & $\begin{array}{c}\text { Personen ohne Aufnahme } \\
\text { einer Beschäftigung }\end{array}$ \\
\hline Mittelwert & 0,680 & 0,352 \\
Standardabweichung & 0,014 & 0,013 \\
T-Test & & $291,97(0,000)$ \\
\hline
\end{tabular}

Quelle: ZEW et al. 2008; Hochrechnung, Berechnungen der Autoren.

WSI MITTELLUNGEN

dass die Aufnahme einer Beschäftigung u. a. auch von den individuellen Dispositionen und Ressourcen abhängt, die im Konzept der individuellen Beschäftigungsfähigkeit zusammengefasst werden. Dies gilt aber nicht für jeden Einzelfall. Bei hochgerechnet 18,0 \% derjenigen Personen, die eine Beschäftigung aufgenommen haben, ist die Beschäftigungsfähigkeit noch unter dem Durchschnitt derjenigen, die beschäftigungslos geblieben sind. Umgekehrt gibt es sogar $25,7 \%$, die beschäftigungslos geblieben sind und deren individuelle Beschäftigungsfähigkeit über dem Durchschnitt der erfolgreichen Wechsler liegt. ${ }^{5}$

\subsection{EINFLUSSFAKTOREN AUF DIE AUFNAHME EINER BESCHÄFTIGUNG}

Für die arbeitsmarktpolitische Diskussion relevant ist die Frage, welche Elemente der Beschäftigungsfähigkeit die relativ wirksamsten sind. Die Einflussfaktoren auf die Aufnahme einer Beschäftigung lassen sich den Ergebnissen der Probit-Schätzung entnehmen, die in Tabelle 2 dargestellt sind. Allerdings enthält diese Tabelle aus Platzgründen nur die Variablen, die die verschiedenen Aspekte von Beschäftigungsfähigkeit abbilden, und nicht die ebenfalls in die Schätzung einbezogenen Kontrollvariablen. Die Kontrollvariablen, insbesondere das Alter, die Arbeitsmarktlage und die regionale Siedlungsstruktur (Landkreis, kreisfreie Stadt) haben einen relativ hohen und robusten Einfluss auf die Wahrscheinlichkeit einer Erwerbsintegration. Allein mit den Kontext- und Kontrollvariablen, also ohne Berücksichtigung von Merkmalen individueller Beschäftigungsfähigkeit, können 5,0 \% der Unterschiede beim Eintritt in Beschäftigung erklärt werden. Durch die Berücksichtigung der Beschäftigungsfähigkeit kann die Erklärungskraft des Modells auf 11,4 \% mehr als verdoppelt werden. Die Kenntnis des Index der Beschäftigungsfähigkeit einer Person verbessert also die Wahrscheinlichkeit einer korrekten Prognose der Beschäfti- gungsaufnahme, aber die Prognose ist weit davon entfernt, sicher zu sein. Dies mag teilweise daran liegen, dass die Operationalisierung von Beschäftigungsfähigkeit gegenüber dem hier präsentierten Index noch verbesserungsfähig ist. Eine vollständige Determinierung der individuellen Beschäftigungsaufnahme durch die gemessene Beschäftigungsfähigkeit würden wir jedoch selbst von einem "optimalen“ Index bei „optimaler“ Kontrolle der Kontextfaktoren des jeweiligen regionalen Arbeitsmarktes nicht erwarten, weil die Beschäftigungsaufnahme auch abhängig ist von Gelegenheitsstrukturen und Veränderungen der individuellen Lebenssituation, deren vollständige Beobachtung statistisch prinzipiell nicht möglich ist. Wegen dieser Prognoseunsicherheit sei davor gewarnt, den Index oder seine Elemente für eine Klassifizierung von ALG-II-Beziehenden nach ihrer „Arbeitsmarktnähe" verwenden zu wollen und Leistungsprozesse im SGB II aufbauend auf der Annahme zu gestalten, dass die Beschäftigungsaufnahme bestimmter Personen von vornherein auszuschließen und bei anderen Personen ein Selbstläufer sei.

$4 \quad$ Als regressionsanalytisches Verfahren wurde die Probit-Schätzung verwendet, da die „abhängige Variable" (Aufnahme einer Beschäftigung) binär ist (ja - eine Beschäftigung wurde aufgenommen; nein - eine Beschäftigung wurde nicht aufgenommen).

5 Die hier und an anderen Stellen dieses Aufsatzes genannten hochgerechneten Werte beziehen sich auf die Grundgesamtheit, die der Stichprobenziehung zugrunde lag. Entsprechend den Anforderungen der Evaluationsstudie (vgl. Abschnitt 3.) wurden ALG-II-Leistungsbeziehende nicht bundesweit befragt, sondern nur im Bereich von 154 Grundsicherungsstellen (die überwiegend Kreisen und kreisfreien Städten entsprechen), die erforderlich waren, um das Regelmodell der Arbeitsgemeinschaft von lokaler Arbeitsagentur und kommunalen Trägern einerseits und das kommunale Optionsmodell andererseits vergleichend zu bewerten. Insbesondere Großstädte sind in der Stichprobe unterrepräsentiert. In den Tendenzen ist jedoch eine gute Übereinstimmung zwischen der "154er Stichprobe“ und dem Bundesgebiet festzustellen. 
rate nur knapp über dem Durchschnitt liegt. Dies deutet für die Männer mit Migrationshintergrund auf eine hohe Beschäftigungsschwelle hin, die nur überwunden werden kann, wenn die individuellen Voraussetzungen für eine Beschäftigungsaufnahme besonders gut sind. Hierzu lässt sich auch die relativ hohe individuelle Beschäftigungsfähigkeit der beschäftigungslos gebliebenen Männer mit Migrationshintergrund heranziehen: Sie konnten ihre vergleichsweise gute Beschäftigungsfähigkeit nicht in eine Beschäftigung umsetzen.

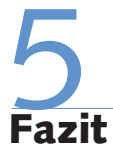

Individuelle Beschäftigungsfähigkeit lässt sich als mehrdimensionales Bündel individueller Eigenschaften verstehen, das die Potenziale einer Person beschreibt, eine Erwerbstätigkeit aufzunehmen, zu behalten oder auszuweiten. Als Dimensionen wurden formale Qualifikationen und Kompetenzen, Gesundheit, Suchverhalten, Konzessionsbereitschaft, Ressourcen zur Arbeitsaufnahme, psycho-soziale Probleme sowie das soziale Umfeld operationalisiert. In diesem Aufsatz wurde ein Konzept vorgeschlagen, demzufolge zunächst der Einfluss dieser Dimensionen auf die Wahrscheinlichkeit einer Beschäftigungsaufnahme im Aggregat empirisch ermittelt wird und anschließend die individuellen Ausprägungen der jeweiligen Dimension mit den geschätzten Einflussstärken gewichtet werden. Dieses Verfahren hat den Vorteil, dass die Erklärungskraft einzelner Komponenten von Beschäftigungsfähigkeit auf Grundlage von tatsächlich erfolgten Arbeitsaufnahmen gemessen wird und dass die Beschäftigungsfähigkeit auch für jene Personen bestimmt werden kann, die keine Arbeit aufgenommen haben. Ein derartiger Indikator ist Voraussetzung, um Veränderungen der individuellen Beschäftigungsfähigkeit („Integrationsfortschritte“) im Zeitverlauf zu untersuchen, auch wenn (noch) keine Beschäftigungsaufnahme erfolgt.

Die empirischen Ergebnisse auf Grundlage einer großen Stichprobe von ALG-IIEmpfängern zeigen:

(1) Die Wahrscheinlichkeit, eine Erwerbstätigkeit aufzunehmen, wird auch unter Kontrolle von soziodemografischen Cha- rakteristika und regionalen Kontextvariablen von den Eigenschaften beeinflusst, die im Konstrukt der individuellen Beschäftigungsfähigkeit operationalisiert werden. Dies ist auch angesichts einerseits wirkmächtiger struktureller, andererseits zahlreicher zufälliger oder unbeobachteter Einflüsse auf Arbeitsmarktchancen und Erwerbsverläufe festzuhalten.

(2) (Ehemalige) ALG-II-Empfänger, die eine Beschäftigung aufgenommen haben, weisen im Durchschnitt eine signifikant höhere Beschäftigungsfähigkeit auf als solche, denen dies nicht gelang. Damit wird eine zentrale theoretische Annahme zum Konstrukt der individuellen Beschäftigungsfähigkeit zum Zusammenhang von individuellen Eigenschaften und Jobchancen empirisch bestätigt.

(3) Besonders hohen Einfluss auf die individuelle Beschäftigungsfähigkeit haben der Gesundheitszustand, die Möglichkeit zu individueller Mobilität (als ein Aspekt der Ressourcen zur Arbeitsaufnahme) sowie formale Qualifikationen. Der Konzessionsbereitschaft kommt hingegen zwar auch ein signifikanter, aber vergleichsweise geringer Stellenwert zu. Die „schlechte Nachricht" für Aktivierungsstrategien: Was durch „Fordern und Fördern" relativ leicht beeinflussbar erscheint, scheint die relativ geringste Wirkung zu haben.

(4) In den meisten der untersuchten Problemgruppen des Arbeitsmarktes gibt es nicht nur geringere Übergangsraten in Beschäftigung, sondern auch unterdurchschnittliche Werte individueller Beschäftigungsfähigkeit für jene, die keine Arbeit aufgenommen haben.

Die Ergebnisse haben aber auch gezeigt, dass die individuelle Beschäftigungsfähigkeit allein den Übergang aus Arbeitslosigkeit in Beschäftigung nicht erklärt. Dies wurde bereits in den Schätzergebnissen der Kontext- und Kontrollvariablen und weiter anhand der Verteilung der Werte individueller Beschäftigungsfähigkeit in verschiedenen Zielgruppen des Arbeitsmarktes deutlich. Ein erheblicher Teil von Personen mit überdurchschnittlicher Beschäftigungsfähigkeit fand keine neue Beschäftigung, während ein nennenswerter Teil von Personen mit unterdurchschnittlicher Beschäftigungsfähigkeit Arbeit gefunden hat. Die hohe Beschäftigungsfähigkeit in eini- gen Zielgruppen (Eltern mit Kindern unter drei Jahren, alleinerziehende Frauen, Männer mit Migrationshintergrund) legt den Schluss nahe, dass arbeitsmarktpolitische Probleme gerade in diesen Gruppen vielfach nicht durch individuelle Faktoren, sondern durch strukturelle Probleme (fehlende Kinderbetreuung, Arbeitsmarktsegmentierung) verursacht sind.

Die Ergebnisse stützen für ALG-IIEmpfänger nicht die Annahme, dass eine fehlende Konzessionsbereitschaft eine wesentliche Ursache für Misserfolge bei der Arbeitsplatzsuche ist. Unsere Befunde legen außerdem nahe, dass zwar faktisch nicht-suchende Leistungsbezieher zu einer Arbeitsuche angehalten werden sollten, dass aber eine breite Streuung der Bewerbungsaktivitäten nicht notwendigerweise schneller in Beschäftigung führt als Unterstützung bei einer zielgenauen Arbeitssuche.

Künftige Forschung zur Beschäftigungsfähigkeit sollte zum einen die einzelnen $\mathrm{Di}$ mensionen des mehrdimensionalen Merkmalbündels „Beschäftigungsfähigkeit“ differenzierter untersuchen. Dies betrifft beispielsweise die Netzwerkdimension, die durch objektive Daten etwa zur Mitgliedschaft in Vereinen erweitert werden kann. Weiterhin sind die Beziehungen der einzelnen Dimensionen untereinander zu berücksichtigen. Damit lassen sich möglicherweise auf den ersten Blick kontraintuitive Befunde, wie der geringe Stellenwert sozialer Problemlagen für eine Beschäftigungsaufnahme, aufklären. Die befragten ALG-II-Beziehenden sind nicht repräsentativ für alle Arbeitslosen. Die Ergebnisse können daher nur vorsichtig verallgemeinert werden. So ist es denkbar, dass eine fehlende Konzessionsbereitschaft unter bestimmten Bedingungen oder in ausgewählten Zielgruppen eine Barriere für eine Beschäftigungsaufnahme darstellt. Die Veränderung der Beschäftigungsfähigkeit unabhängig von einer Beschäftigungsaufnahme ist eine weitere Forschungsfrage von praktischer Relevanz; das hier vorgestellte Konzept erlaubt einen Vorher-Nachher-Vergleich der Beschäftigungsaufnahme auch von Personen, die keine Beschäftigung aufgenommen haben. In jedem Fall eröffnet die hier vorgenommene Operationalisierung individueller Beschäftigungsfähigkeit neue Perspektiven, um die Diskussionen zum Stellenwert von Beschäftigungsfähigkeit auf empirischer Grundlage zu führen. 


\section{LITERATUR}

Apel, H. (2006): Beschäftigungsfähigkeit und soziale Stabilisierung. Ein Überblick über Inhalt und Operationalisierung, Unveröffentlichtes Manuskript, Köln

Apel, H./Fertig, M. (2009): Operationalisierung von „Beschäftigungsfähigkeit“" Ein methodischer Beitrag zur Entwicklung eines Messkonzepts, in: Zeitschrift für Arbeitsmarktforschung 1, S. 5-28

Bellmann, L./Kistler, E./Wahse, J. (2007): Demographischer Wandel: Betriebe müssen sich auf alternde Belegschaften einstellen, IAB-Kurzbericht 21, Nürnberg

Blancke, S./Roth, Ch./ Schmid, J. (2000): Employability („Beschäftigungsfähigkeit“) als Herausforderung für den Arbeitsmarkt. Auf dem Weg zur flexiblen Erwerbsgesellschaft. Eine Konzept- und Literaturstudie, Akademie für Technikfolgenabschätzung, Stuttgart

Deeke, A./Kruppe, Th. (2003): Beschäftigungsfähigkeit als Evaluationsmaßstab? Inhaltliche und methodische Aspekte der Wirkungsanalyse beruflicher Weiterbildung im Rahmen des ESF-BA-Programms, IAB-Werkstattbericht 01, Nürnberg

Europäische Kommission (1997): Die beschäftigungspolitischen Leitlinien für 1998. Entschließung des Rates vom 15. Dezember 1997. Online verfügbar unter http://ec.europa.eu/employment_social/employment_ strategy/98_guidelines_de.htm
Gazier, B. (1998): Employability - definitions and trends, in: Gazier, B. (Hrsg.): Employability. Concepts and policies, Berlin, S. 37-71 Lefresne, F. (1999): Employability at the heart of the European employment strategy, in: Transfer 4, S. 460-480

McQuaid, R. W./Lindsay, C. (2005): The concept of employability, in: Urban Studies 2, S. 197-219

Promberger, M./Wenzel, U./Pfeiffer, S./Hacket, A./Hirseland, A. (2008) Beschäftigungsfähigkeit, Arbeitsvermögen und Arbeitslosigkeit, in: WSIMitteilungen 2, S. 70-76

Söstra /IMU /PIW /Compass (2005): Evaluation der Maßnahmen zur Umsetzung der Vorschläge der Hartz-Kommission. Modul 1c: Arbeitsbeschaffungsmaßnahmen. Zweiter Bericht, Berlin

ZEW /IAQ /TNS Emnid (2008): Evaluation der Experimentierklausel nach § 6c SGB II - Vergleichende Evaluation des arbeitsmarktpolitischen Erfolgs der Modelle der Aufgabenwahrnehmung "Optierende Kommune " und "Arbeitsgemeinschaft". Untersuchungsfeld 3: „Wirkungs- und Effizienzanalyse" , Abschlussbericht, Mannheim 